\title{
Assessment of Drug Utilisation Evaluation of Cephalosporins in A Tertiary Care Hospital
}

\author{
Parvathy Rajan $\mathbf{P}^{1}$, Priscilla Mary ${ }^{2}$, Sheik Haja Sherief ${ }^{2}$, Sivakumar $\mathbf{T}^{\mathbf{3}}$ \\ 1Pharm D. Intern, Department of Pharmacy Practice, Nandha College of Pharmacy, Erode, Tamilnadu, India. \\ 2 Department of Pharmacy Practice, Nandha College of Pharmacy, Erode, Tamilnadu, India. \\ ${ }^{3}$ Principal, Nandha College of Pharmacy, Erode, Tamilnadu, India. \\ *Corresponding author's E-mail: parvathyrajan622@gmail.com
}

Received: 18-12-2020; Revised: 06-04-2021; Accepted: 13-04-2021; Published on: 15-05-2021.

\begin{abstract}
Drug Use Analysis (DUR) or Drug Use Assessment (DUE) is known as an important quality management method for evaluating and improving substance use and patient care. It is an ongoing, systematic method to examine the pattern of drug administration in a number of clinical environments, with recommendations or defined criteria to facilitate the acceptable and efficient use of drugs. The DUE can be organized for evaluation and to improve the method of prescribing or dispensing the medication according to acceptable indications, dosage, drug reactions, therapeutic efficacy and cost-effectiveness. The aim of this study to evaluate the drug utilization pattern of cephalosporins. A retrospective study was performed for a period of 2 months among the hospitalized patients who received cephalosporins for different indications in which 100 patients were enrolled. Inappropriate use of drug therapy has been found in phase 1 (pre- intervention) and interventions have been conducted to rationalize the appropriate use of drugs in terms of indication, dose, dose length, individualized dosage modification, which has been strengthened in phase 2 by providing effective guidance on the application of DUE and interventions.
\end{abstract}

Keywords: Drug Utilization Review, Drug Use Evaluation, Rational use of drug, Intervention.

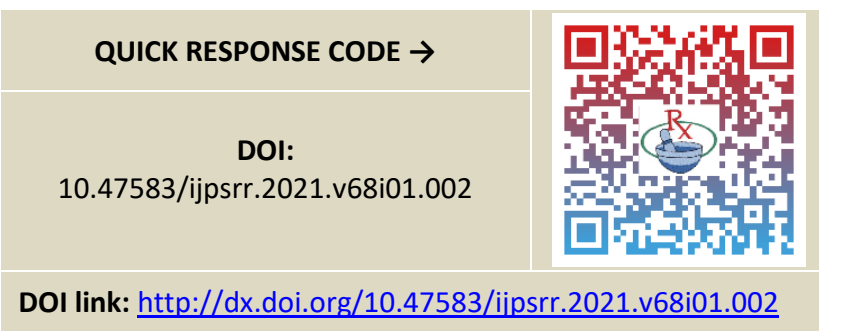

INTRODUCTION

ntimicrobial resistance has emerged as a significant health care quality and patient safety issue due to the improper usage of antibiotics, increased number of medications and treatment options which, resulted in an irrational usage of antibiotics leading to a poor patient outcome.

Therefore, it was decided to evaluate the current utilization pattern of cephalosporins among different patients to rationalize drug usage in accordance with specific indications, dose, dosage regimen and individualized dosage regimen. So, Drug utilization evaluation (DUE) has been recommended as a method for identifying inappropriate or unnecessary drug use in order to promote rational therapy. Growing concern over the widespread misuse of antibiotics together with the emergence of antimicrobial resistance and escalating expenditures has resulted in antibiotics being the drugs most frequently chosen for DUE projects, by which these guidelines could be utilized effectively for the improvement of drug usage, overuse, misuse, and abuse. ${ }^{1}$
Drug Utilization Evaluation (DUE) is an ongoing, authorized and systemic quality improvement process, which is designed to Review drug use and prescribing patterns, provide feedback of results to clinicians and other relevant groups, develop criteria and standards which describes optimal drug use, promote appropriate drug use through education and other interventions. ${ }^{2}$

Cephalosporins are primarily used as betalactam agents, which are preferable over other antibiotics dueto their lo wer hypersensitivity reactions, an objective wide range of action, lower cost and better outcome, having given its advantages, it is important to maintain the sensitivity of Cephalosporin. Although Cephalosporin use also offers lifesaving treatment to those who have a severe bacterial infection, resistance to antimicrobials is a global problem and some of the major global threats are multi-drug resistant tuberculosis and drug resistant malaria. ${ }^{3}$

A DUE will -

- Identify acceptable use of medicinal products according to accepted guidelines.

- Audit to evaluate the prescribing trend based on the parameters

- Provide guidance to prescribers after review of dependent treatments and modifications.

Track to see whether the guidelines are being met and the prescribing pattern. ${ }^{4}$ 


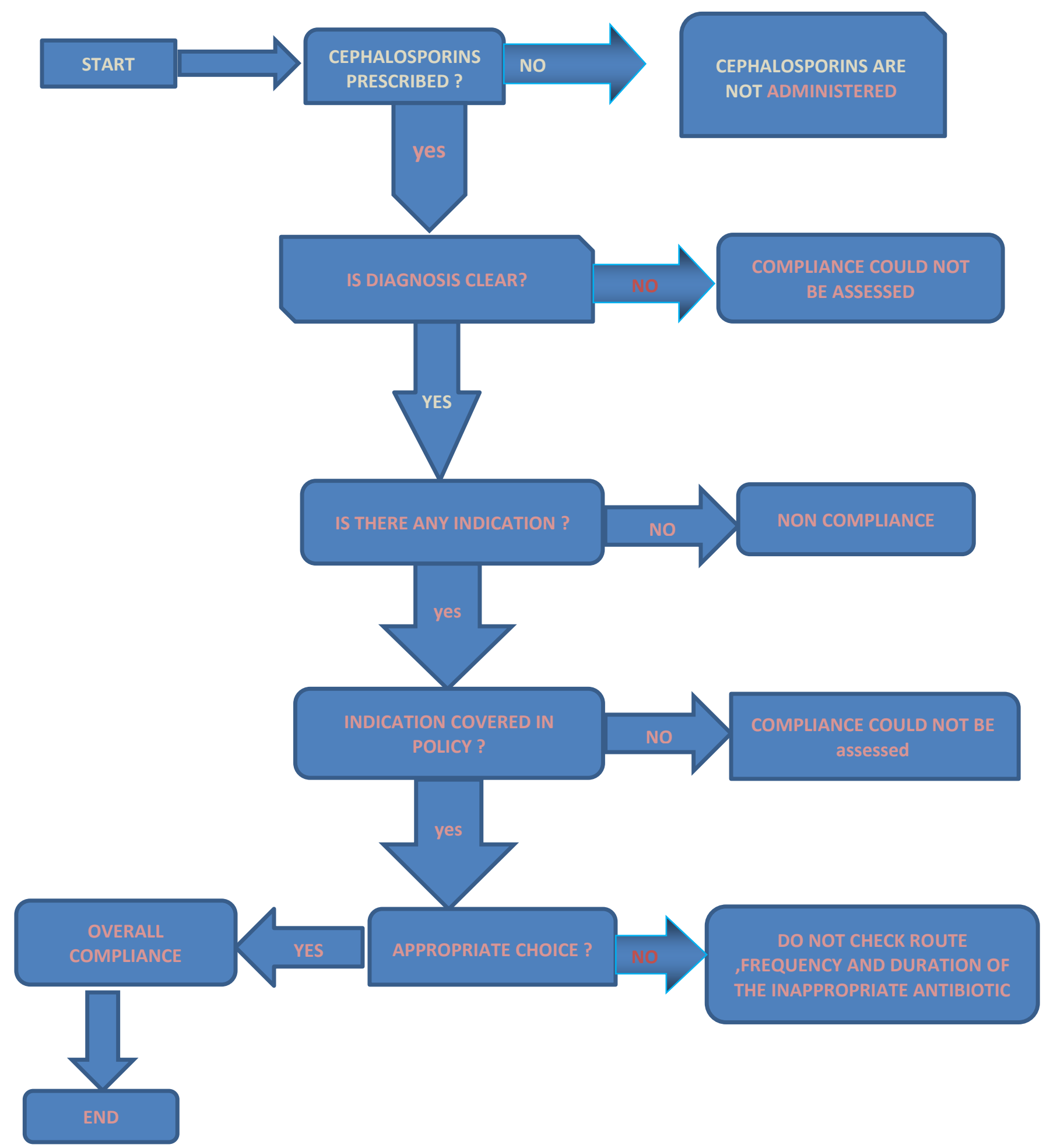

A DUE framework can be developed in a short period of time, after it has become apparent, it will track the issues there seems to be in the use of medicines. Many of these issues may be established from other DUE research, an analysis of aggregate hospital data (e.g., most expensive medications, most prescribed drugs, ADR records, medical chart reports, hospital, or guidelines from DTC (Drug Therapeutic committee) members. Daily Drug therapeutic committee meetings and quality control reviews in the health care sector should be able to recognize issues that can be addressed in a DUE for resolution. . $^{5}$
Clinical pharmacist will play an important role in the design and execution of the DUE guidelines.

1. Encouraging multidisciplinary cooperation within the health care system to ensure a sound DUE programme.

2. Help for adequate choice of medications, optimum dosing, rapid initiation and careful monitoring.

3. 3.To support the goals and priorities of the DUE.

4. Monitor policies on antimicrobial usage by partnering with pharmacies and other similar mechanisms to 
ensure microbiological patterns within the health care system.

5. Generalize and interpret quantitative data.

6. To document outcomes of the program to perform clinical and economic analysis.

7. Presentation of the DUE findings obtained at meetings and conferences.

8. To educate the health care team about and use of DUE

\section{METHODS}

The DUE programme included educational intervention and regulatory intervention to address trends of drug use that did not comply with agreed standards and requirements, followed by the second step of the audit to assess the efficacy of the intervention in subsequent prescriptions.

\section{Objectives}

To evaluate the drug utilisation of cephalosporins.

\section{Study criteria}

\section{Inclusion Criteria}

All patients receiving cephalosporins irrespective of age, gender, type of infection, the average number of drugs per prescription were included in the study.

\section{Exclusion criteria}

Those who are not taking cephalosporins in their prescription were excluded from the study.

\section{Source of data}

Patient data related to the study was obtained from the following sources and recorded in the well-designed patient data collection form:

- Treatment chart/case sheet, lab report, hospital antibiogram

- Patient or patient's care giver interview.

\section{Study procedure}

Patient enrolment

A retrospective study was conducted. The information was collected from patient case sheet, prescribed with Cephalosporins for a period of 2 months. A whole data of patient's case note was selected from inpatient medical record and the information obtained from each patient case note was transferred into a well-designed data collection form

\section{Determination of prescribing pattern}

Well-designed data collection form was prepared and required information was extracted from inpatient's information sheet which included:
Patient Demographic: Name, age, gender, weight, blood pressure, personal history, medication history.

Drug data: which includes name of the drug, dosage regimen, dose and route of anti-microbial agent (AMA), AMAs per patient, duration of therapy

\section{Statistical method}

The information collected regarding all the selected cases were recorded in the data collection form and the percentage was calculated

\section{RESULTS AND DISCUSSION}

A total of 100 cases were obtained for study purposes. The study was divided into 2 stages, phase 1 as prior to the intervention in which the project charter was created, including the title, clinical need, aim and scope of the project. Following the identification, the data collection method was created to collect data from patient case sheets, antibiotics and laboratory charts. Then moved to phase 2, following the intervention and implementation of the proposed action, where another 50 cases were collected and the enforcement of the DUE policies was analysed. We tend to decrease the irrational usage of cephalosporins and increased the efficacy of treatment and quality of life.

Sample size $: 100$

Among the 100 patients enrolled in the study as shown in $48 \%$ were males and $52 \%$ were females.

\section{Age Distribution in Two Phases}

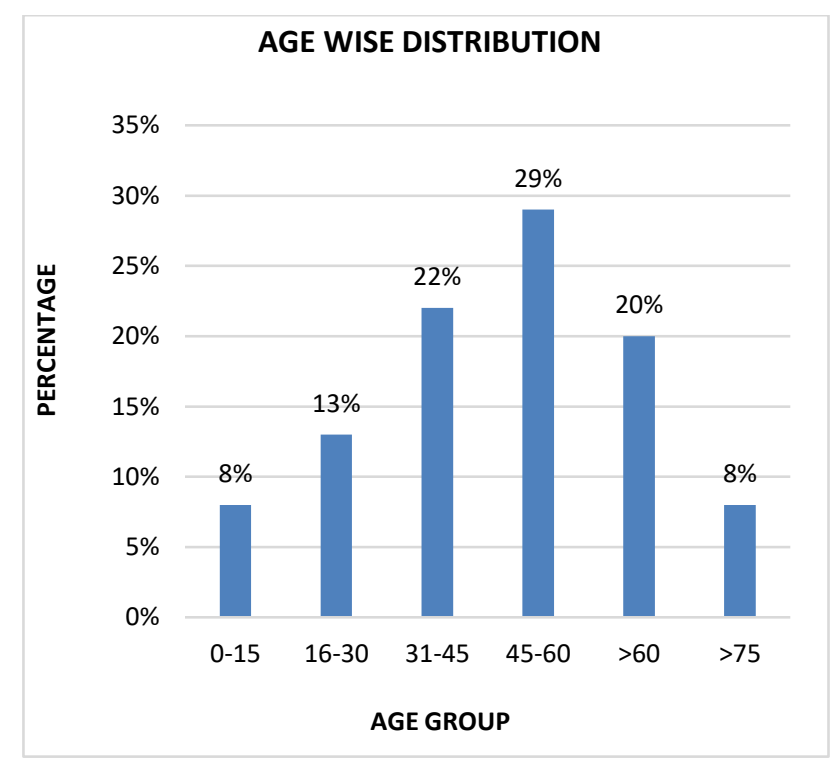

Figure 2: Age Distribution

Among 100 patients enrolled in the study, as shown in (Figure 2) Patients in between age group of 45-60 y were more predominantly prescribed with cephalosporins in both phases. 


\section{Weight Distribution of Patients in Both Phases}

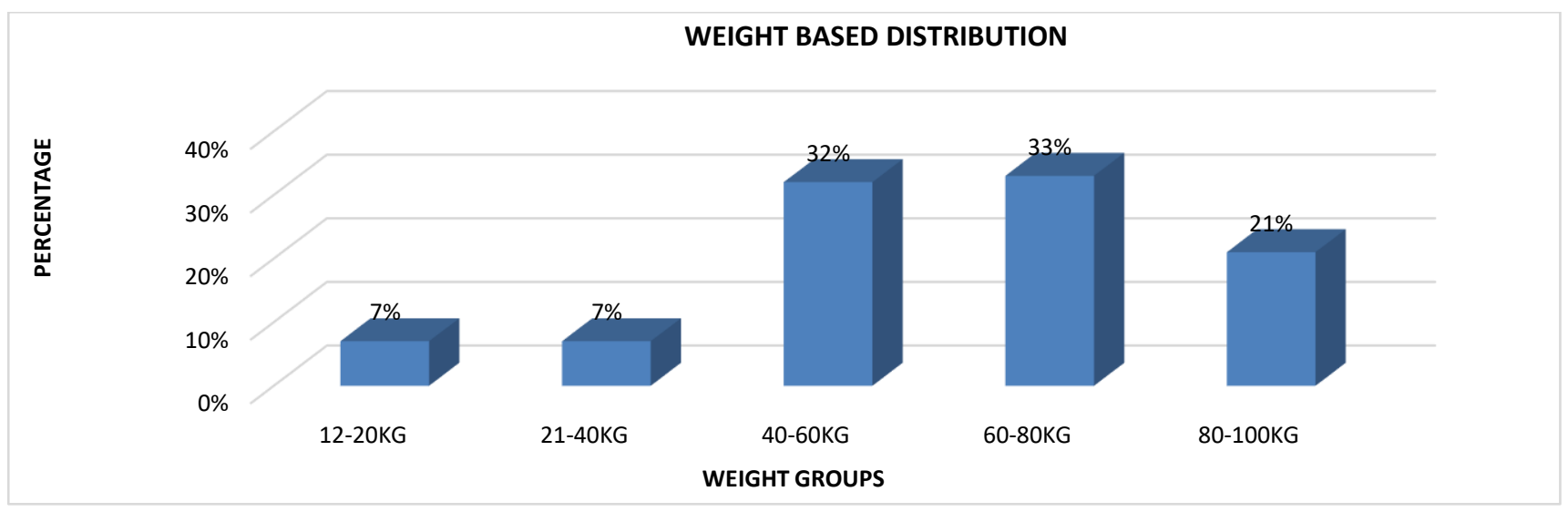

Figure 3:

Among 100 patients enrolled in the study, as shown in Figure 3 patients who used cephalosporins were more in number among ( $60-80 \mathrm{~kg}$ )and (40 -60 kg)

Utilization of cephalosporins in both phases.

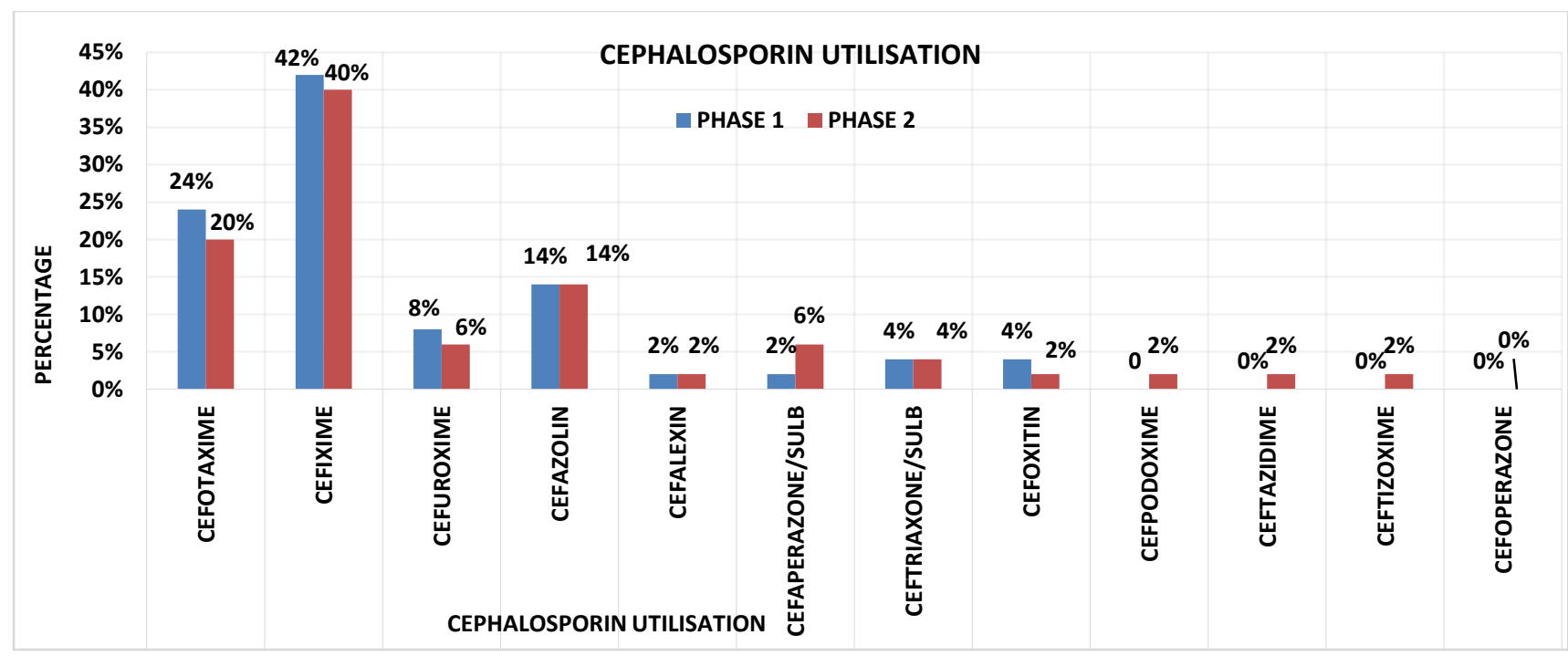

Figure 3:

Among 100 patients enrolled in the study, as shown in Figure 3,most commonle used antibiotic was cefotaxime40\% in phase 1 and $42 \%$ in phase 2 respectively, followed by ceftriaxone $24 \%$ in phase 1 and $20 \%$ in phase 2 respectively, cefuroxime $14 \%$ in both phases, cefixime $6 \%$ in phase 1 and $8 \%$ in phase 2 respectively, combination of cefaperazone/sulbactam as $4 \%$ in both phases and ceftriaxone/sulbactam as $4 \%$ in phase 1 and $2 \%$ in phase 2 .

\section{Based On Prescribed Indications}

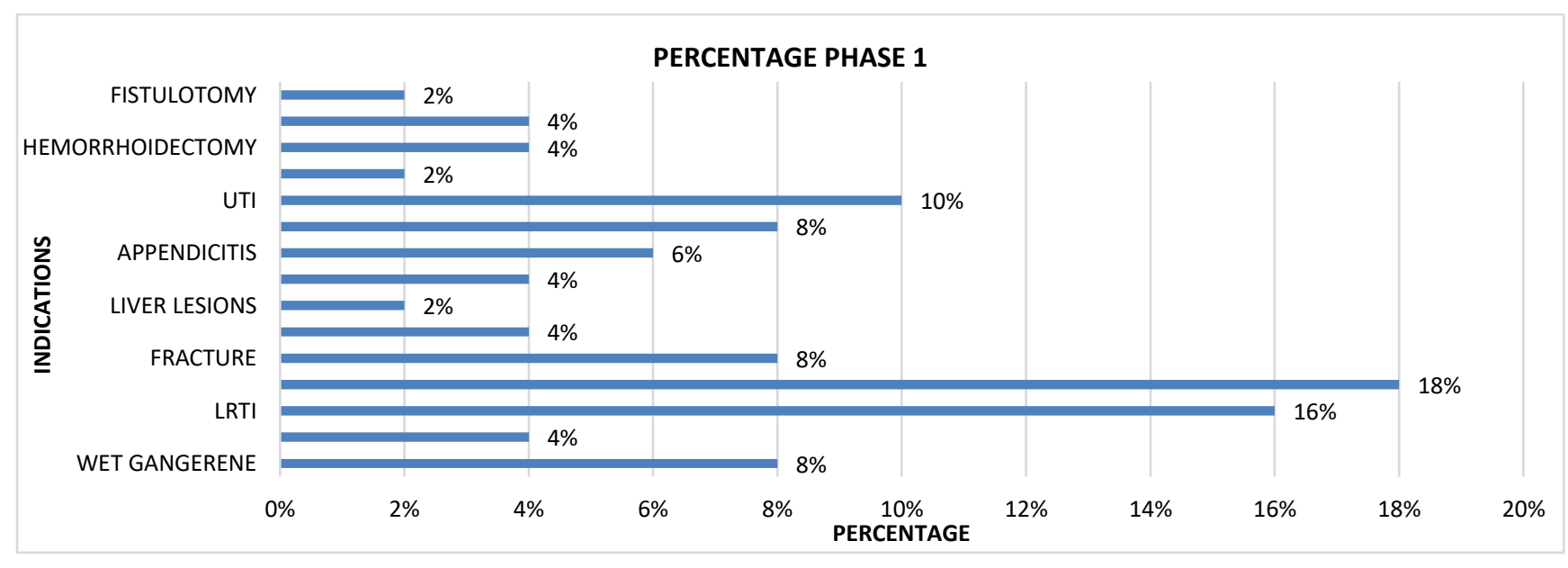




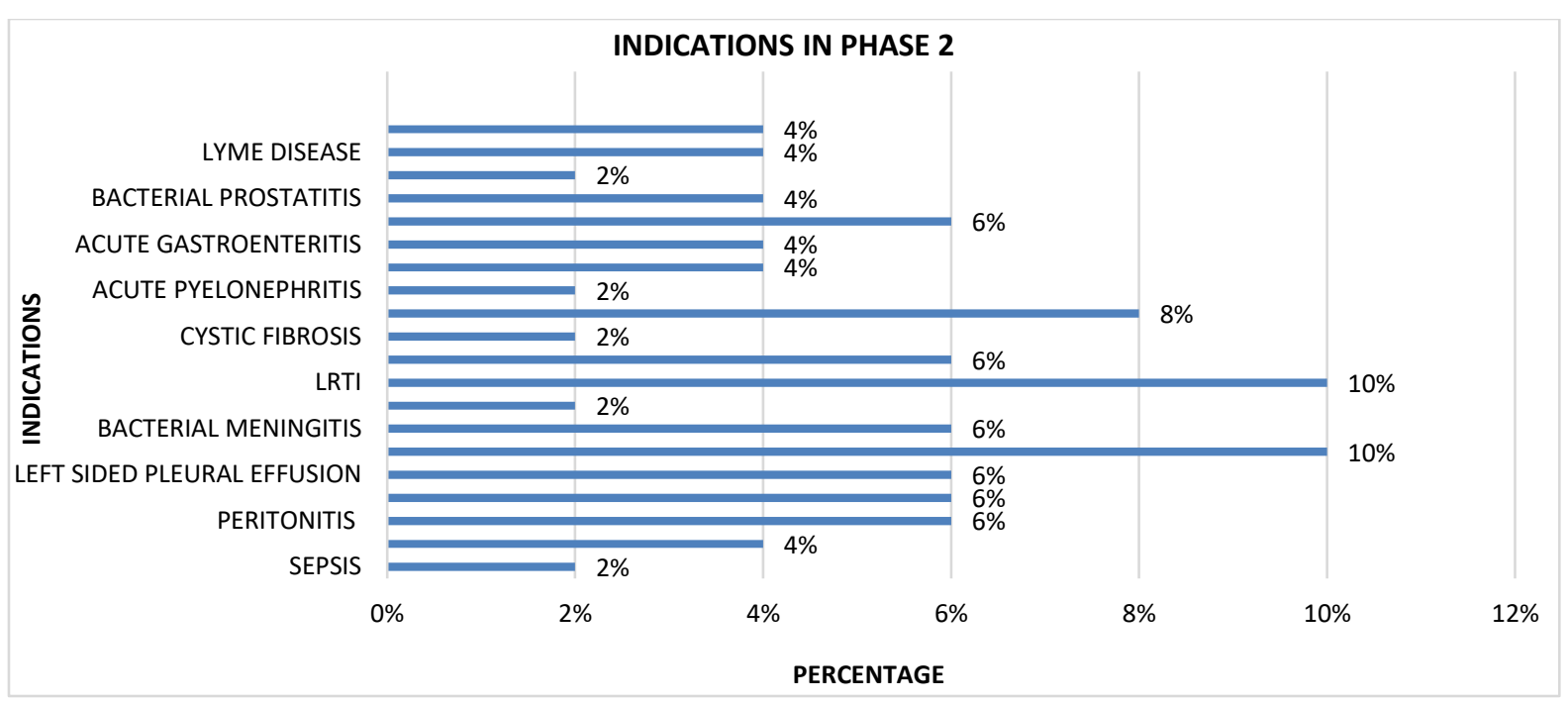

\section{Figure 4: \& Figure 5:}

Among 100 patients enrolled in the study, as shown in Figure 4,5 number of patients who were prescribed rationally for their indication has increased in phase 2 When compared to phase 1.

The administration of cefoperazone / sulbactam combined with azithromycin to treat children with lobar pneumonia has shown positive results and may be used as the first option of treatment for lobar pneumonia. Cefoperazone / sulbactam, which has strong antibacterial properties, contains an inhibitor of $\beta$-lactamase called sulbactam, while azithromycin has been shown to have important therapeutic effects on infections caused by Mycoplasma pneumoniae, Haemophilus influenzae and Streptococcus pneumoniae, which makes this combination drug highly effective. ${ }^{(6)}$

Extended-spectrum cephalosporin optimises the outcome of Klebsiella Pneumoniae, related liver abscess. It has greater penetration in the aqueous humour of the eye, the cerebrospinal fluid, the synovial fluid, and the pericardial fluid. ${ }^{(7)}$

Aerobic gram-negative bacilli are the primary pathogens in bacterial prostatitis. Most antibiotics enter acutely inflamed prostate but evidence suggests about treatment with a broad-spectrum beta-lactam drug; either penicillin (e.g. piperacillin-tazobactam) or cephalosporin (e.g. cefotaxime or ceftazidim) perhaps combined with aminoglycoside in patients who are seriously ill. ${ }^{(8)}$

The treatment of gangrene generally includes broad spectrum antibiotics such as penicillin G, clindamycin, vancomycin, and gentamicin which are used as empiric therapy in case of methicillin-resistant Staphylococcus aureus (MRSA), gram-negative, gram-positive, and anaerobic microbes. ${ }^{(9)}$ The combination of ceftazidime plus glycopeptide is superior to other regimens for the initial treatment of Peritoneal dialysis associated peritonitis, caused by enteric gram-negative rods such as Escherichia coli and Klebsiella species (10) Spontaneous bacterial peritonitis is a prevalent disease in patients with cirrhosis related ascites, even though it occurs so often ,its discovered only accidentally when paracentesis is performed. The ascitic fluid acquired by paracentesis cytologic analysis remains the gold standard for diagnosis for SBP. Enteric gram-negative rods and streptococci make up the preponderance of SBP pathogens. Management of SBP consists of several antibiotic options, including cefotaxime and ceftriaxone. Patients should be evaluated after 48 hours to determine whether expanded antibiotic therapy is warranted. (11)

Cephalexin, Cefdinir, Cefprozil and Cefaclor are cephalosporins that treat Staphylococcus aureus infections and Cefuroxime is used for Pseudomonas aeruginosa infections, which are the common drug resistant infections in those with cystic fibrosis. ${ }^{(12)}$

Ceftazidime has been successfully used as a single agent in Pseudomonas aeruginosa infections. Because of its broad spectrum and its activity against penicillin-resistant and aminoglycoside-resistant Pseudomonas, Ceftazidime can be used empirically as a single agent instead of combinations in patients with cystic fibrosis. ${ }^{(13)}$

In case of Catheter associated infections, Vancomycin is usually recommended when there is an incidence of methicillin-resistant staphylococci, because of its activity against coagulase-negative staphylococci and Streptococcus aureus, but as an add-on empiric coverage for enteric gram-negative bacilli and Pseudomonas aeruginosa with use of a third or fourth generation cephalosporins such as ceftazidime or cefepime, in case of severely ill or immunocompromised patients who have suspected catheter-related bloodstream infection. ${ }^{(14)}$

Most cephalosporins distribute well into the extracellular fluid of most tissues, and some of the later-generation cephalosporins also sufficiently penetrate into cerebrospinal fluid to be used in the treatment of central nervous system infections Third and fourth generation cephalosporins typically have activity against salmonellae 
and therefore could be used for the management of enteric fever (15)

\section{Culture Report Data}

Phase 1

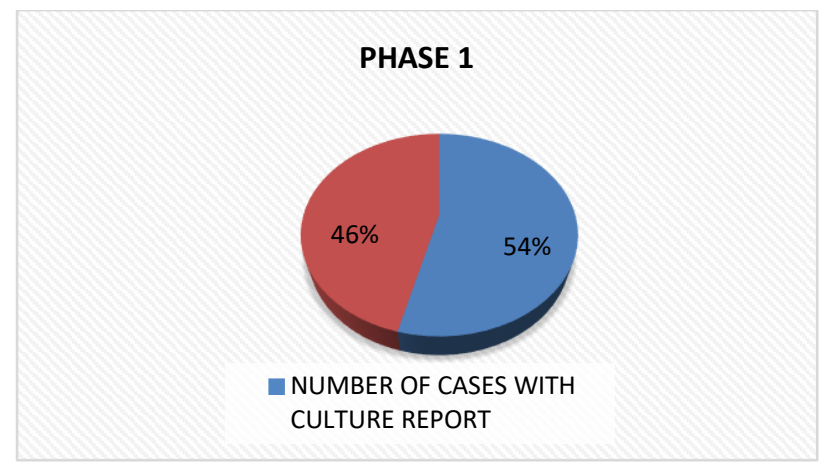

Figure 6:

\section{PHASE 2}

Distribution Based On Culture Growth

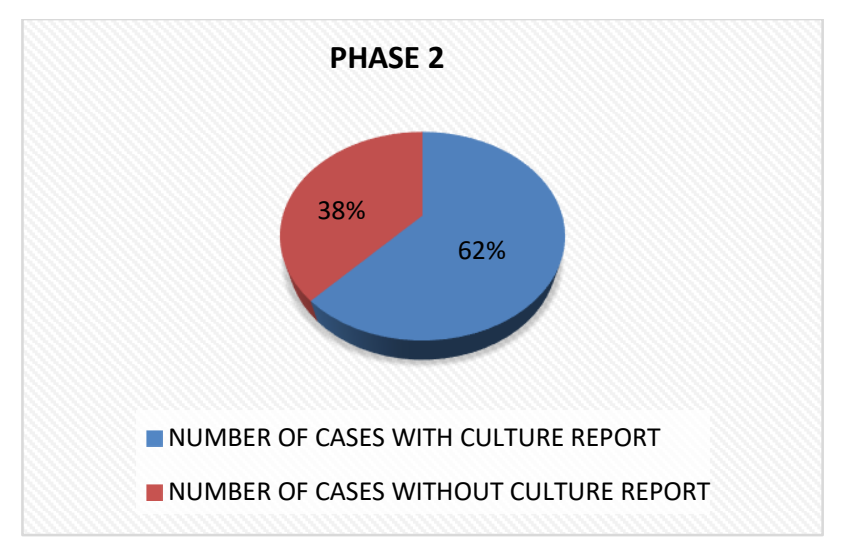

Out of 100 cases collected in phase $1,54 \%$ of cases were found to be with culture report and $46 \%$ of cases were found to be without culture report and this has got significantly optimised to $38 \%$ of cases without culture report and $62 \%$ of cases with culture report in phase 2

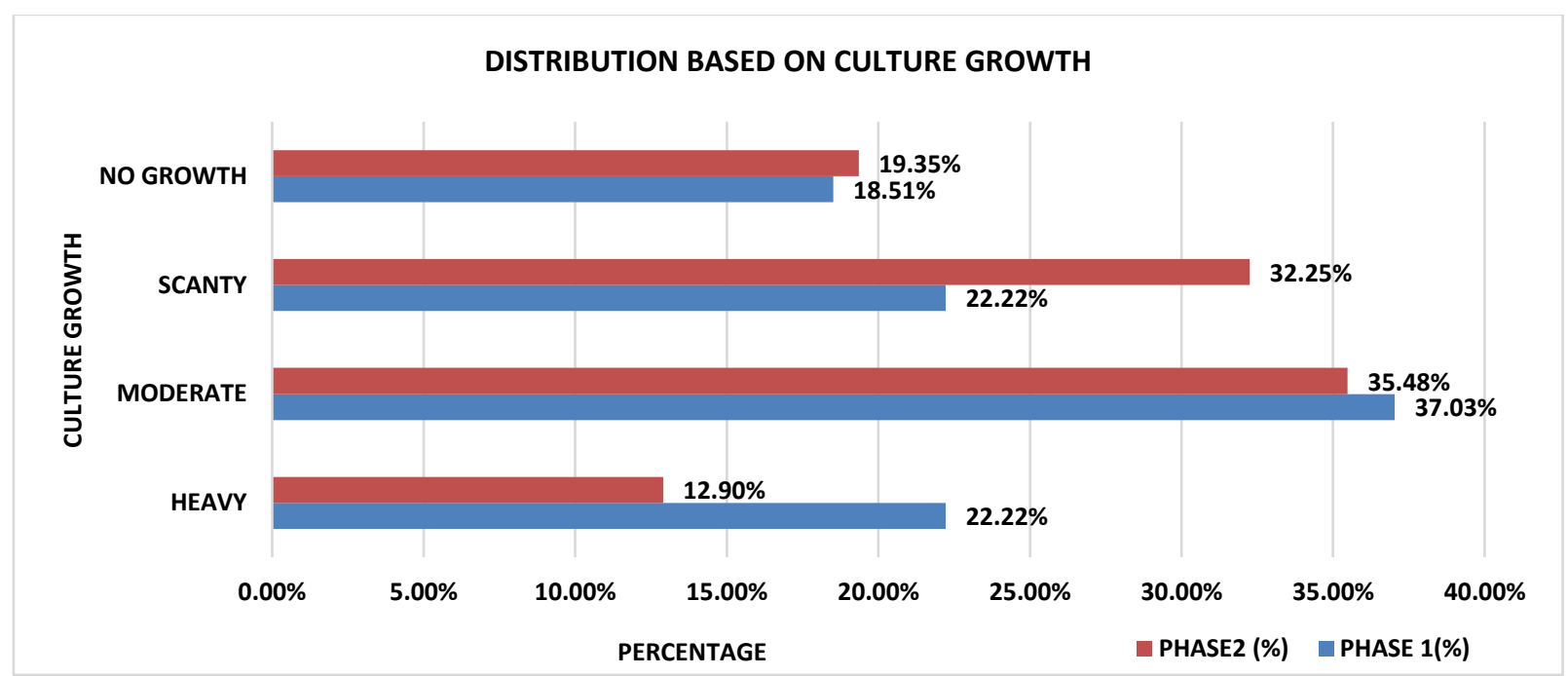

Figure 9:

The growth pattern of microorganisms isolated were classified into scanty, moderate heavy and no growth. In phase 2 of our study the growth of our microorganism was scanty because of the optimum use of antibiotics

\section{Distribution Based On Microorganism}

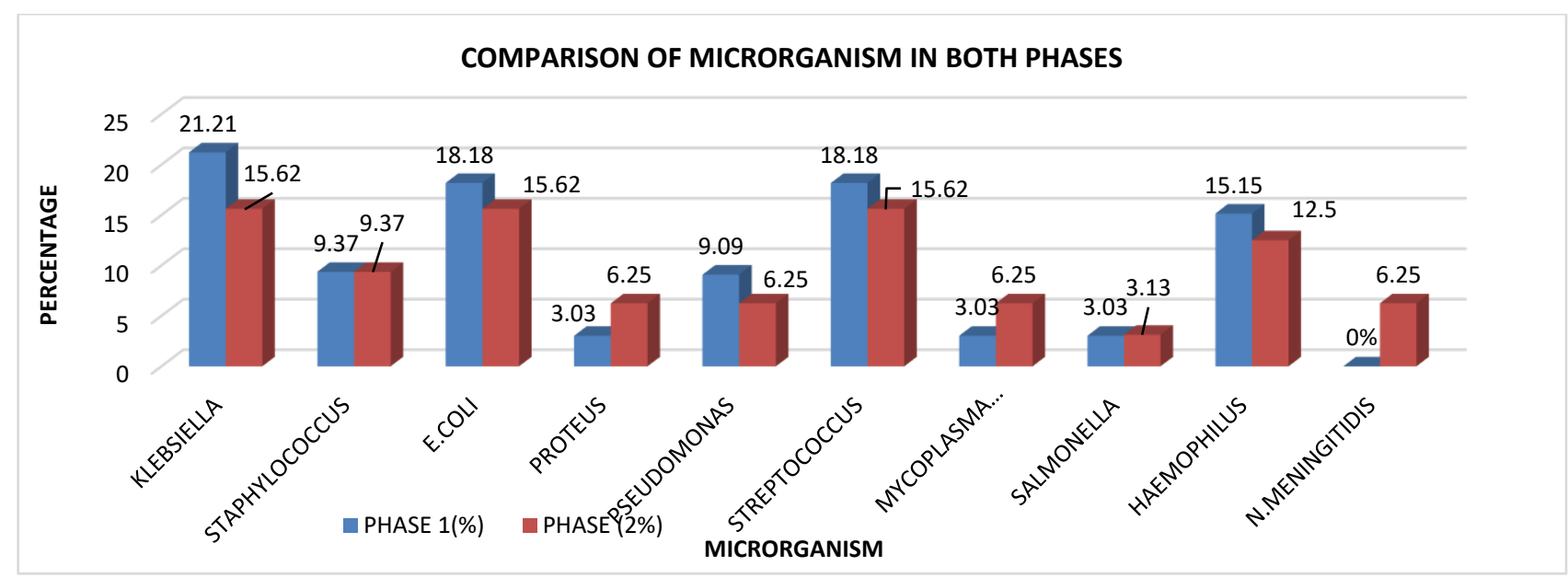

Figure 10 
Microorganisms in culture report was identified that $21.21 \%$ of growth is for klebsiella species, $18.18 \%$ of growth for E.coli, streptococcus species, $15.15 \%$ of growth for haemophilus species in phase 1 and in phase 2 it has been decreased to $15.62 \%$ of Klebsiella species, $15.62 \%$ of growth for E.coli, streptococcus species, $12.5 \%$ of growth for haemophilus species, $6.25 \%$ of growth for mycoplasma pneumoniae. meningitidis species was seen respectively which was susceptible to first or second generation of cephalosporins and higher usage of antibiotics was spared.

\section{Antibiotic Utilization Pattern}

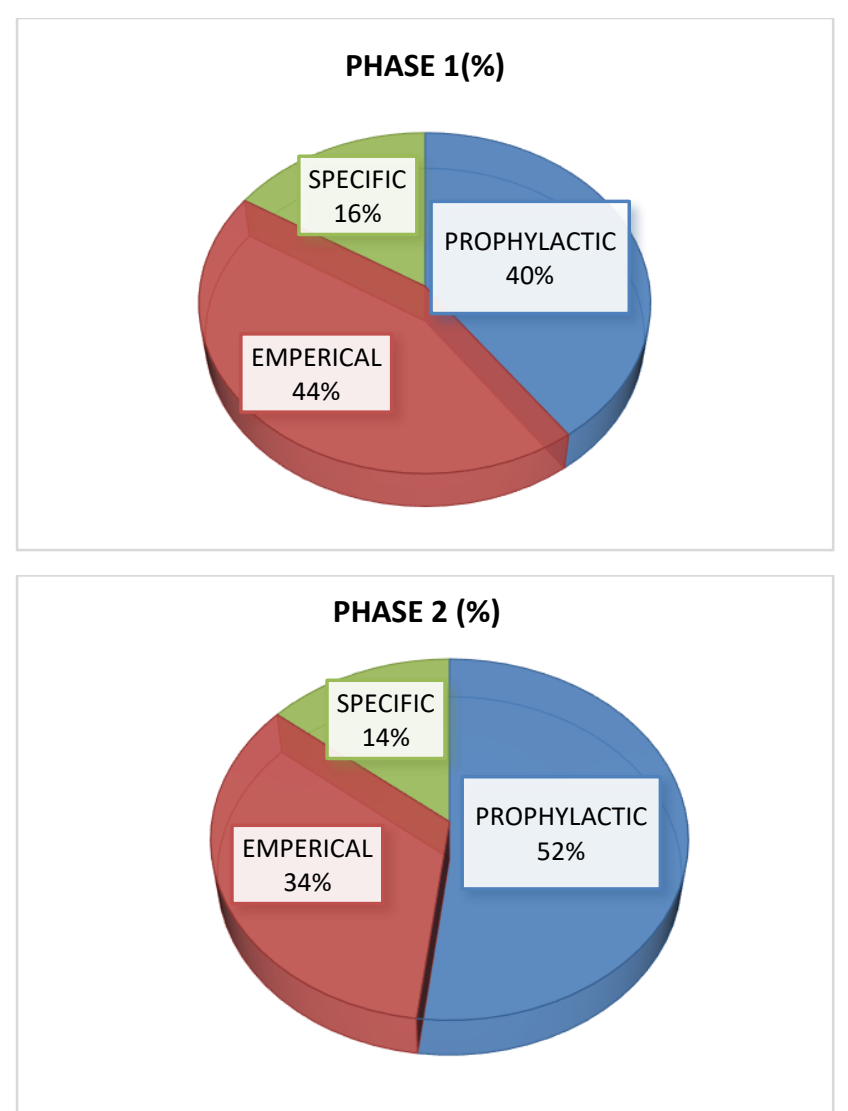

On basis of cephalosporin utilization pattern, prophylactic usage of antibiotic was enhanced by $40 \%$ in phase 1 to $52 \%$ in phase 2. Empirical therapy was more in phase I than in phase 2 and prescribing for actual treatment was low in phase 1 which was intervened while carrying phase 2 and an appropriate prescribing pattern for pathogen specific therapy was focused.

\section{Drug Interactions Observed In Two Phases}

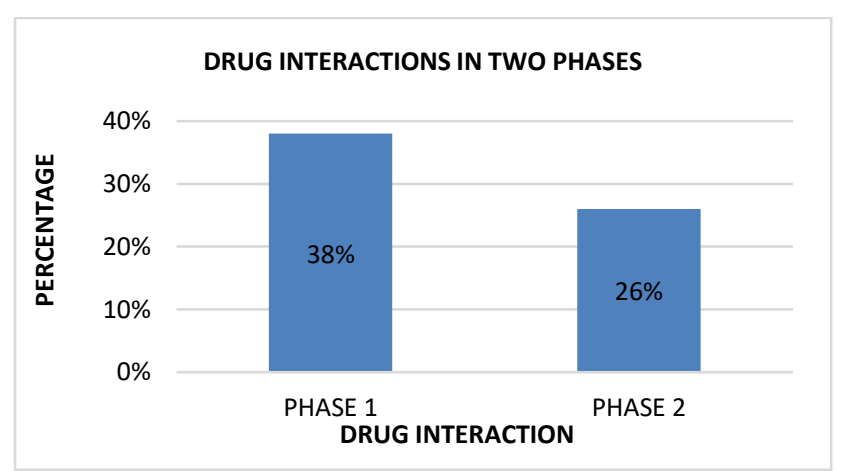

Figure 10:
Among 100 patients enrolled in the study as shown in fig10 and, number of drug interactions has decreased from $38 \%$ in phase 1 to $26 \%$ in phase 2

Thus, in conclusion, Phase 1 comprised of identification of patients with cephalosporin therapy followed by documentation of their data their data including culture reports, antibiograms, and Indications. After the analysis interventions were established and intervened policies of standard guidelines concerning the utilization of antibiotics were implemented. In phase 2 an analysis if these implemented strategies were assessed and the improvement in treatment efficacy and quality of the utilization of cephalosporins were monitored

The most common indication for use of Cephalosporin was respiratory tract infections, whereas in the General Surgery, wound debridement was the most common indication. Cefotaxime was the most commonly prescribed cephalosporin in general medicine and cefoperazone/sulbactam combination drug was found effective in pathogens like Mycoplasma pneumoniae, Haemophilus influenzae and Streptococcus pneumoniae, an extended-spectrum cephalosporin. Other empiric usage of cephalosporin was justified during second phase based upon the conditions of the patient and the need for individualization.

\section{CONCLUSION}

Cephalosporins are the most widely used form of antibiotics that require close supervision to ensure safe usage at this time because there is an increased risk of microbial resistance. The key purpose and difficulty of antibiotic policies is the careful evaluation of the condition of the patient. Correct prescription pattern of antibiotic by the physician and Patients' adherence to proper drugs is integral. DUE strategy will help avoid microbial resistance, minimize the costs and increase the quality of patient care. It reduces the burden of use of unnecessary antibiotics and increases pathogen-oriented treatment. As the drug or host-related response pertaining to antibiotic therapy can lead to treatment failure and excessive use of antibiotics could lead to an increase in the cost of care, resulting in financial loss due to increased length of stay in hospital patients.

\section{REFERENCES}

1. Misan GM, Dollman C, Shaw DR, Burgess N. Cephalosporin utilisation review and evaluation. Pharmacoeconomics.; 1995; 8(2): 100-22.

2. John SM, Panda BK, Bhosle DG, Soman N. Evaluation of cephalosporins utilization and compliance with reference to the hospital antibiotic policy of an Indian tertiary care hospital. International Journal of Basic \& Clinical Pharmacology.; 2019; 8(5): 1044.

3. Cosgrove SE, Kaye KS, Eliopoulous GM, Carmeli Y. Health and economic outcomes of the emergence of thirdgeneration cephalosporin resistance in Enterobacter species. Archives of internal medicine.; 2002; 162(2): 18590. 
4. John SM, Panda BK, Bhosle DG, Soman N. Evaluation of cephalosporins utilization and compliance with reference to the hospital antibiotic policy of an Indian tertiary care hospital. International Journal of Basic \& Clinical Pharmacology ; 2019; 8(5): 1044.

5. Bisht R, Bhattacharya S, Katiyar A. Utilization of Third Generation Cephalosporins in Multispeciality Teaching Hospital, Dehradun. Indian Journal of Pharmacy Practice; 2009; 2(3).

6. Li Y, Han F, Yang Y, Chu J. Principles of antibiotic application in children with lobar pneumonia: Step-up or step-down. Experimental and therapeutic medicine; $2017 ; 13(6)$ : 26814.

7. Cheng HP, Siu LK, Chang FY. Extended-spectrum cephalosporin compared to cefazolin for treatment of Klebsiella pneumoniae-caused liver abscess. Antimicrobial agents and chemotherapy; 2003; 47(7): 2088-92.

8. Lipsky BA, Byren I, Hoey CT. Treatment of bacterial prostatitis. Clinical Infectious Diseases.; 2010; 50(12): 1641-52.
9. Haylee Boyens, Wet gangrene, Dermnet NZ. 2014.

10. Barretti P, Doles JV, Pinotti DG, El Dib R. Efficacy of antibiotic therapy for peritoneal dialysis-associated peritonitis: a proportional meta-analysis. BMC infectious diseases; 2014; 14(1): 445.

11. Alaniz C, Regal RE. Spontaneous bacterial peritonitis: a review of treatment options. Pharmacy and Therapeutics; 2009; 34(4): 204.

12. Cephalosporins for Infections in Cystic Fibrosis, Cytsic fibrosis new today Smith BR. Cefsulodin and ceftazidime, two antipseudomonal cephalosporins. Clinical Pharmacy.; 1984; 3(4): 373-85.

13. Mermel LA, Farr BM, Sherertz RJ, Raad II, O'Grady N, Harris JS, Craven DE. Guidelines for the management of intravascular catheter-related infections. Clinical infectious diseases; 2001; 32(9): 1249-72.

14. Stoesser N, Eyre D, Basnyat B, Parry C. Treatment of enteric fever (typhoid and paratyphoid fever) with third and fourth generation cephalosporins. Cochrane Database of Systematic Reviews. 2013; 8(3):52-59.

Source of Support: None declared.

Conflict of Interest: None declared.

For any question relates to this article, please reach us at: editor@globalresearchonline.net New manuscripts for publication can be submitted at: submit@globalresearchonline.net and submit_ijpsrr@rediffmail.com 